\title{
Non-canonical Fzd7 signaling contributes to breast cancer mesenchymal-like stemness involving Col6a1
}

\author{
Ping Yin ${ }^{1 \dagger}$, Yu Bai ${ }^{1,2+}$, Zhuo Wang ${ }^{1}$, Yu Sun ${ }^{1}$, Jian Gao ${ }^{1}$, Lei Na ${ }^{1}$, Zhongbo Zhang ${ }^{1}$, Wei Wang ${ }^{1 *}$ and \\ Chenghai Zhao ${ }^{1 *}$ iD
}

\begin{abstract}
Mesenchymal-like stemness is characterized by epithelial-mesenchymal transition (EMT). Breast cancer (BC) cell mesenchymal-like stemness is responsible for distal lung metastasis. Interrogation of databases showed that Fzd7 was closely associated with a panel of mesenchymal-related genes and a panel of stemness-related genes. Fzd7 knockdown in mesenchymal-like MDA-MB-231 and Hs578T cells reduced expression of Vimentin, Slug and Zeb1, induced an epithelial-like morphology, inhibited cell motility, impaired mammosphere formation and decreased $\mathrm{Lgr}^{+}$subpopulation. In contrast, Fzd7 overexpression in MCF7 cells resulted in opposite changes. Fzd7 knockdown delayed xenograft tumor formation, suppressed tumor growth, and impaired lung metastasis. Mechanistically, Fzd7 combined with Wnt5a/b and modulated expression of phosphorylated Stat3 (p-STAT3), Smad3 and Yes-associated protein 1 (Yap1). Moreover, Fzd7-Wnt5b modulated expression of collagen, type VI, alpha 1 (Col6a1). Both Wnt5b knockdown and Col6a1 knockdown disrupted BC cell mesenchymal phenotype and stemness. Taken together, Fzd7 contributes to BC cell EMT and stemness, inducing tumorigenesis and metastasis, mainly through a non-canonical Wnt5b pathway. Col6a1 is implicated in Fzd7-Wnt5b signaling, and mediates Fzd7-Wnt5b -induced mesenchymallike stemness.
\end{abstract}

Keywords: Frizzled 7, Stemness, Breast cancer, Lgr5, Wnt

\section{Introduction}

Frizzled (Fzd) are seven-transmembrane proteins harboring a conserved 120-amino-acid cysteine-rich domain (CRD), via which Fzd combine with extracellular Wnt molecules. Wnt/Fzd pair initiates $\beta$-catenin -dependent or independent signaling pathways. Wnt/ $\beta$-catenin is one of the most important oncogenic pathways involved in many aspects of tumors such as proliferation, epithelial-mesenchymal transition (EMT), angiogenesis, chemoresistance, stemness and metastasis. Signaling

\footnotetext{
*Correspondence: wangwei07@cmu.edu.cn; chzhao@cmu.edu.cn

${ }^{\dagger}$ Ping Yin and Yu Bai contributed equally to this work.

'Department of Pathophysiology, College of Basic Medical Science, China Medical University, Shenyang, China

Full list of author information is available at the end of the article
}

initiated by Wnt molecules such as Wnt5a and Wnt5b, is independent on $\beta$-catenin, and conventionally called non-canonical Wnt pathway. Actually, non-canonical Wnt pathway is also related to EMT, stemness and metastasis [1-3].

There are 10 members identified in human Fzd family. Fzd7 is implicated in human cancers. Fzd7 was upregulated in hepatocellular carcinoma (HCC), resulting in activation of $\beta$-catenin pathway, accompanied by increased TCF transcriptional activity and cell proliferation rate [4-6]. Fzd7/ $\beta$-catenin pathway was also activated in triple-negative breast cancer (TNBC), responsible for cell proliferation $[7,8]$. However, recent studies demonstrated that Fzd7 also mediated non-canonical Wnt signaling. Wnt5a-Fzd7 pair promoted growth and tumorigenesis of

(c) The Author(s). 2020 Open Access This article is licensed under a Creative Commons Attribution 4.0 International License, which permits use, sharing, adaptation, distribution and reproduction in any medium or format, as long as you give appropriate credit to the original author(s) and the source, provide a link to the Creative Commons licence, and indicate if changes were made. The images or other third party material in this article are included in the article's Creative Commons licence, unless indicated otherwise in a credit line to the material. If material is not included in the article's Creative Commons licence and your intended use is not permitted by statutory regulation or exceeds the permitted use, you will need to obtain permission directly from the copyright holder. To view a copy of this licence, visit http://creativecommons.org/licenses/by/4.0/ The Creative Commons Public Domain Dedication waiver (http://creativecommons.org/publicdomain/zero/1.0/) applies to the data made available in this article, unless otherwise stated in a credit line to the data. 
melanoma [9]. Moreover, Fzd7 signaling induced gastric tumorigenesis irrespective of APC mutation status, suggesting this process might not be related to $\beta$-catenin [10].

Cancer cell stemness is related to distal metastasis, relapse and chemoresistance. Mesenchymal-like stemness is characterized by EMT [11]. EMT has been shown to induce mammary stem cell and tumor-initiating cell stemness $[12,13]$. Oncogenic pathways such as IL-6/ Stat3, EGFR, NOTCH and TGF- $\beta 1$ are involved in breast cancer (BC) cell mesenchymal phenotype and stemness [14-17]. Interrogation of databases indicated that Fzd7-mediated non-canonical Wnt pathway crosstalked with these pathways, thereby suggesting Fzd7 might contribute to $\mathrm{BC}$ cell mesenchymal-like stemness. Interrogation of databases further identified Wnt5b as a potential ligand for Fzd7, and collagen, type VI, alpha 1 (Col6a1) as a downstream molecule in Fzd7-Wnt5b signaling.

\section{Methods}

In silico analysis

Cancer Cell Line Encyclopedia (CCLE) database and GSE12777 database were interrogated for gene expression in human BC cell lines. GSE2603 database was interrogated for gene expression in human $\mathrm{BC}$ tissues. Correlation between two genes was analyzed by Pearson statistics. Heat maps were generated by GraphPad Prism 7.0.

\section{Cell culture}

MDA-MB-231, Hs578T, MCF7 and BT-549 cells were cultured in DMEM with $10 \%$ fetal bovine serum (FBS) and $1 \%$ penicillin/streptomycin at $37{ }^{\circ} \mathrm{C}$ and $5 \% \mathrm{CO} 2$ in a humidified incubator. T47D cells were cultured in RPMI1640 with $10 \%$ fetal bovine serum (FBS) and 1\% penicillin/streptomycin. All cell lines were obtained from ATCC.

\section{Western blot}

Cells were washed twice with cold PBS and were lysed in RIPA buffer with Phenylmethanesulfonyl fluoride (both Beyotime). BCA Protein Assay Kit (Beyotime) was used to quantify protein concentrations. Proteins were separated by SDS-PAGE and transferred onto PVDF membranes (Immobilon-P). 5\% bovine serum albumin was performed to block membranes. Primary antibodies include Fzd7 (Santa Cruz Biotechnology, sc-293,261), Col6al (Santa Cruz Biotechnology, sc-377,143), Wnt5a/b (Cell Signaling Technology, \#2530), IL-6 (Cell Signaling Technology, \#12153), p-Stat3 (Cell Signaling Technology, \#9145), E-cadherin (Cell Signaling Technology, \#3195), Vimentin (Cell Signaling Technology, \#5741), Slug (Cell Signaling Technology, \#9585), Zeb1 (Sigma, SAB2102759), Smad3 (Cell Signaling Technology, \#9523), Yap1 (Cell
Signaling Technology, \#14074), CD44 (Abcam, ab51037) and GAPDH (Proteintech, HRP-60004). Membranes were incubated with primary antibody overnight, and with horseradish peroxidase-conjugated secondary antibody for $2 \mathrm{~h}$. SuperSignal Chemiluminescent Substrates (Thermo Fisher Scientific) and imaging systems were used to analysis the results.

\section{Real-time PCR}

RNAiso Plus (Takara) was used to extract total RNAs, which were then reversely transcribed into cDNA using PrimeScript $^{\text {tw }}$ RT reagent Kit with gDNA Eraser (TaKaRa) according to the instructions. Real-time PCR was performed using TB Green ${ }^{\mathrm{Tx}}$ Premix Ex Taq ${ }^{\mathrm{Th}}$ II (TaKaRa). Primers for VIM (Vimentin) were, forward: 5'-GGTGGACCAGCTAACCAACG-3' and reverse: 5' TTGCAGGGTGTTTTCGGCTT-3'; for CDH1 (E-cadherin) were, forward: 5'-GCCATCGCTTACACCATC CTCAG-3' and reverse: 5'-CTCTCTCGGTCCAGCC CAGTG-3'; for ZEB1 were, forward: 5'-CAGGCAAA GTAAATATCCCTGC-3' and reverse: 5'-GGTAAAAC TGGGGAGTTAGTCA-3'; for SNAI2 (Slug) were, forward: 5' -CTGTGACAAGGAATATGTGAGC-3' and reverse: 5'-CTAATGTGTCCTTGAAGCAACC-3'. GAPDH was used as endogenous control. Expression difference was analyzed using $2^{-\triangle \Delta C T}$ method.

\section{Cell transfection}

$\mathrm{BC}$ cells were transfected with shRNA lentiviruses, and selected with $2 \mu \mathrm{g} / \mathrm{ml}$ puromycin (Invitrogen) $48 \mathrm{~h}$ posttransfection. Cells with gene stable knockdown were maintained in DMEM supplemented with 10\% FBS and $2 \mu \mathrm{g} / \mathrm{ml}$ puromycin. MCF7 cells were transfected with FZD7 overexpression lentiviruses and selected with $1 \mu \mathrm{g} /$ $\mathrm{ml}$ puromycin. Cells with FZD7 stable overexpression were cultured in DMEM contained with 10\% FBS and $1 \mu \mathrm{g} / \mathrm{ml}$ puromycin.

\section{Immunofluorescence assay}

Cells were grown on cover slips for $24 \mathrm{~h}$, then washed twice with PBS and fixed by $4 \%$ paraformaldehyde solution. Cytomembranes were penetrated with $1 \%$ Triton100 solution. Primary antibodies (Cell Signaling Technology, E-cadherin, \#3195; Vimentin, \#5741) were added at $4{ }^{\circ} \mathrm{C}$ overnight. Cells were then incubated with Alexa fluor 488donkey anti-rabbit $\operatorname{IgG}(\mathrm{H}+\mathrm{L})$ (Invitrogen, A21206) for $2 \mathrm{~h}$, and with DAPI staining solution (Beyotime) for $5 \mathrm{~min}$ at room temperature in the dark. The fluorescence was visualized by a confocal microscope.

\section{Wound healing assay}

Cells were cultured in six-well plate. A pipette tip was used to scratch confluent monolayers. Cell debris was washed away with warm PBS. Then cells were cultured 
for $24 \mathrm{~h}$ in serum-free medium. An inverted microscope was used to capture the images.

\section{Cell invasion assay}

Cells were resuspended with serum-free medium and made into $1 \times 10^{5} / \mathrm{ml} .0 .2 \mathrm{ml}$ of the cell suspensions $(2 \times$ $10^{4}$ cells) were added to the upper chamber of transwell plates (Corning Costar). In the lower chamber, $0.5 \mathrm{ml}$ medium with $10 \%$ FBS was added to promote cell movement through the pores of the membrane. After $24 \mathrm{~h}$, the cotton swab was used to clean the inside of the chamber. Migrated cells were fixed with a paraformaldehyde solution for $15 \mathrm{~min}$ and stained with the Crystal Violet. Images were captured using an inverted microscope.

\section{Mammosphere assay}

$1 \times 10^{4}$ cells were seeded in 6-well Ultra-Low Attachment Surface Polystyrene culture plates (Corning Costar) added with complete MammoCult ${ }^{\mathrm{tm}}$ Human Medium (STEMCE LL Technologies). Cells were cultured at $37^{\circ} \mathrm{C}$ and $5 \%$ $\mathrm{CO}_{2}$ for 8 days. Analysis was performed by counting the number of mammospheres in 5 randomly selected fields under an inverted microscope.

\section{Flow cytometry}

The proportion of $\mathrm{Lgr}^{+}$stem cells was determined by flowcytometry. Pre-cooled stain buffer (Invitrogen) was used to wash and resuspend cells. $1 \times 10^{6}$ cells were stained with $5 \mu \mathrm{l} \mathrm{PE}$-conjugated anti-hLgr5 (Invitrogen) antibody on ice for $30 \mathrm{~min}$. Stained populations were sorted and analyzed on BD Accuri C6 Plus (BD Biosciences).

\section{In vivo study}

All animal experiments were performed using 8-weekold NOD SCID mice. Mice were maintained in laminar flow rooms with constant temperature and humidity. $5 \times 10^{5}$ MDA-MB-231 cells transfected with FZD7 lentiviruses or control lentiviruses were injected into the fat pad of mice. Tumor growth was followed every 7 days by tumor diameter measurements using vernier calipers. Tumor volumes (V) were calculated using $1 / 2 \times$ length $\times$ width $^{2}$. Ten weeks after inoculation, tumors and lungs were obtained, and fixed in $4 \%$ paraformaldehyde solution. Lung samples were stained with HE and images were captured by using a positive microscope. All animal experiments were approved by the ethics committee of the China Medical University.

\section{Immunohistochemistry}

Immunohistochemistry (IHC) analysis was performed on $4 \mu \mathrm{m}$ paraffin sections of human BC tissues and mouse xenograft tumors. Xylene and gradient alcohols were performed to deparaffinize and hydrate, respectively. 3\%
$\mathrm{H}_{2} \mathrm{O}_{2}$ was added to the sections to remove endogenous peroxidase. Sections were incubated with citrate buffer to repair antigen, and blocked by BSA. Primary antibodies were used as follows: Fzd7 (Santa Cruz Biotechnology, sc293,261), Col6a1 (Santa Cruz Biotechnology, sc-377,143), Ki67 (Invitrogen, 14,569,982), Cleaved caspase-3 (Cell Signaling Technology, \#9661). After incubated with primary antibodies overnight at $4{ }^{\circ} \mathrm{C}$ in a wet box, biotinylated secondary antibodies were added. Diaminobenzidine (BOSTER) was used to stain the sections dissolved in Tris- $\mathrm{HCl}$ and $\mathrm{H}_{2} \mathrm{O}_{2}$. Then sections were re-stained in hematoxylin, dehydrated with gradient alcohol and xylene and sealed with cover slides.

\section{Human specimens}

Paraffin-embedded BC tissues including 10 invasive ductal BC (IDC), 5 invasive lobular BC (ILC) and 3 ductal BC in situ (DCIS) were obtained from Liaoning Province Tumor Hospital with the informed consent of the patients. The use of these specimens for research purposes was approved by Institutional Research Ethics Committee of China Medical University.

\section{Co-immunoprecipitation}

Cell lysates of MDA-MB-231 and Hs578T were centrifuged at $12000 \mathrm{rpm}$ at $4{ }^{\circ} \mathrm{C}$. Fifty microliter supernatant was taken as normal sample and $20 \mu \mathrm{l}$ protein A/G agarose beads (Santa Cruz Biotechnology, sc-2003) were added to the remaining supernatant and incubated for $30 \mathrm{~min}$. After incubating and centrifuging, samples were divided into two tubes. Two microgram Fzd7 (R\&D systems) and $2 \mu \mathrm{g}$ mouse IgG (Santa Cruz Biotechnology) were added, respectively. Each tube sample was combined with $20 \mu \mathrm{l}$ protein $\mathrm{A} / \mathrm{G}$ agarose beads overnight. The non-specifically bound proteins were removed by washing the agarose beads. Following procedures were same as Western blot.

\section{Statistical analysis}

GraphPad Prism 7.0 was used to analyze the data, and all data were presented as the mean \pm s.e.m. Comparison of means within two groups was analyzed using twotailed unpaired Student's $t$ test. $P<0.05$ was considered as significant.

\section{Results}

\section{Fzd7 is associated with mesenchymal phenotype}

To investigate the association of Fzd7 with BC cell mesenchymal phenotype, we first interrogated Cancer Cell Line Encyclopedia (CCLE) database and GSE12777 database. It was shown that expression of FZD7 was positively correlated with that of mesenchymal-related genes, whereas negatively correlated with that of epithelial-related genes, in human BC cell lines (Fig. 1a-b, Supplementary file 1: 


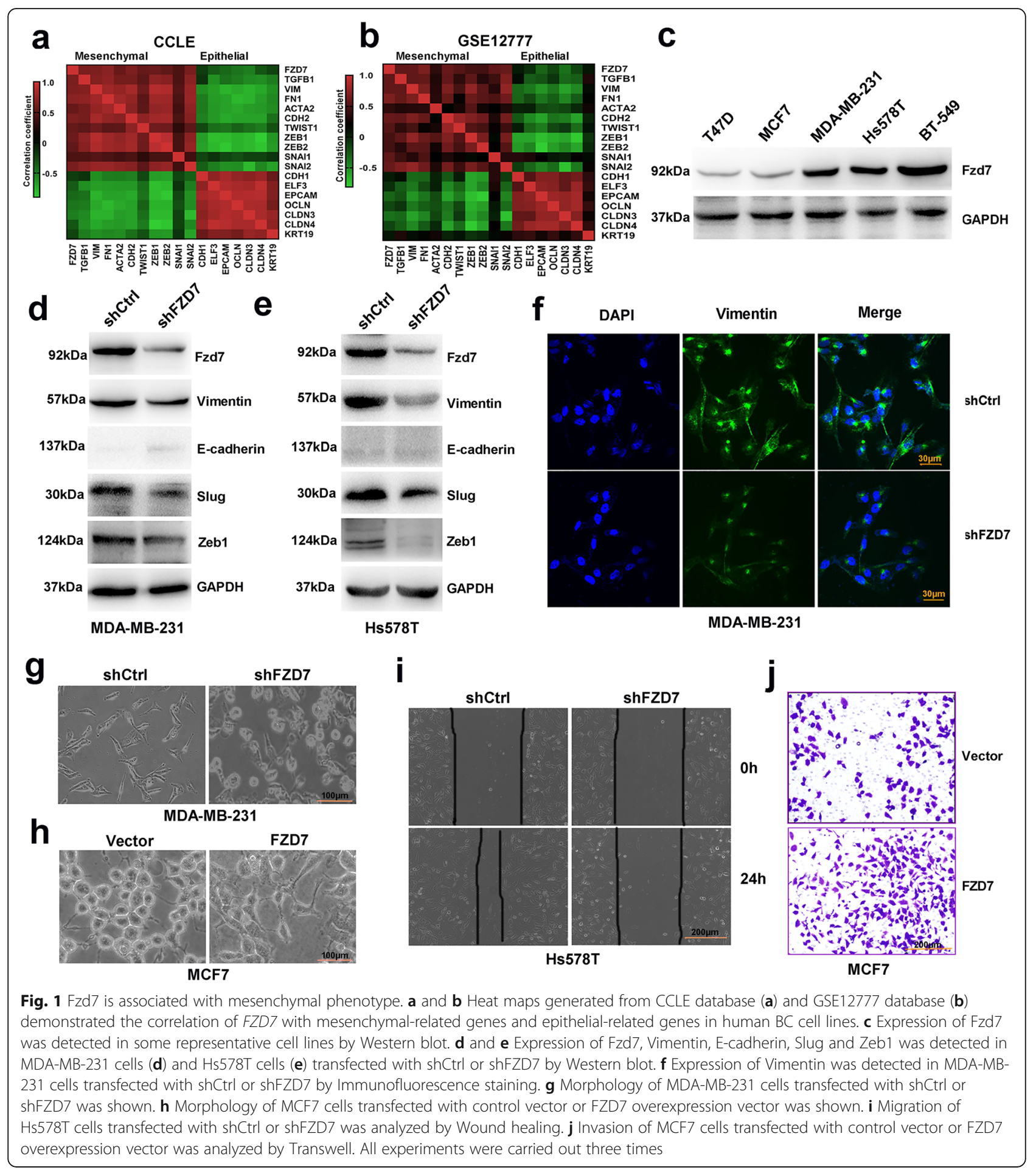

Fig. S1A-B). Expression of Fzd7 in some representative cell lines was subsequently determined. As shown by Western blot detection, Fzd7 was highly expressed in several mesenchymal-like cell lines such as MDA-MB-231, Hs578T and BT-549 (Fig. 1c).

To further explore the role of Fzd7 in BC cell mesenchymal phenotype, MDA-MB-231 cells and Hs578T cells were transfected with FZD7 shRNA lentiviruses. Fzd7 knockdown significantly reduced expression of Vimentin, N-cadherin and EMT transcription factor (EMT-TF) Slug and Zeb1 (Fig. 1d-e, Supplementary file 1: Fig. S1C-F). The inhibitory effect of Fzd7 knockdown on expression of Vimentin was also confirmed by Immunofluorescent staining (Fig. 1f). Consistently, Fzd7 
overexpression in MCF7 cells upregulated expression of Vimentin, Slug and Zeb1 (Supplementary file 1: Fig. S2A-C). Fzd7 knockdown induced an epithelial-like morphology in MDA-MB-231 cells, while Fzd7 overexpression induced a mesenchymal-like morphology in MCF7 cells (Fig. 1g-h). Moreover, Fzd7 knockdown suppressed $\mathrm{BC}$ cell migration and invasion, whereas Fzd7 overexpression promoted BC cell motility (Fig. 1i-j, Supplementary file 1: Fig. 3a-d).

\section{Fzd7 contributes to BC cell stemness}

To explore whether Fzd7 was related to BC cell stemness, we interrogated CCLE database, and found a correlation of FZD7 with a panel of stemness-related genes including CD44, LGR5, NOTCH2, EGFR, IL6 as well as TNC [18] and ANTXR1 [19] (Fig. 2a, Supplementary file 1: Fig. S4A). Interrogation of GSE12777 revealed that FZD7 was significantly correlated with CD44, NOTCH2, EGFR, IL6 and ANTXR1 (Fig. 2b, Supplementary file 1: Fig. S4B). Moreover, interrogation of GSE2603 indicated that FZD7 was correlated with LGR5, NOTCH2, EGFR and $T N C$ in a panel of human BC tissues (Fig. 2c, Supplementary file 1: Fig. S4C).

Mammosphere formation test was then used to evaluate the role of Fzd7 in BC cell stemness. Fzd7 knockdown impaired mammosphere formation capacity of MDA-MB-231 cells and Hs578T cells, while Fzd7 overexpression promoted mammosphere formation in MCF7 cells (Fig. 2d-e, Supplementary file 1: Fig. S5A). Furthermore, Fzd7 knockdown reduced the fraction of $\operatorname{Lgr} 5^{+}$ subpopulation in MDA-MB-231 cells and Hs578T cells, whereas Fzd7 overexpression increased the fraction of $\mathrm{Lgr}^{+}$subpopulation in MCF7 cells (Fig. 2f-g, Supplementary file 1: Fig. S5B). Loss and gain of Fzd7 also affected CD44 expression in BC cells (Supplementary file 1: Fig. S5C-D). Moreover, Fzd7 knockdown had no effect on the subpopulation of aldehyde dehydrogenase 1 (ALDH1) which was recognized as a marker of epithelial-like stemness [11] (data not shown).

\section{Fzd7 knockdown suppresses BC tumorigenesis and metastasis}

To evaluate the effect of Fzd7 knockdown on BC tumorigenesis and metastasis in vivo, NOD SCID mice were inoculated with MDA-MB-231 cells transfected with FZD7 shRNA lentiviruses or control shRNA lentiviruses. Xenograft tumors were harvested and lung metastasis was analyzed 10 weeks after inoculation. Tumors derived from FZD7 shRNA-transfected cells grew much slower than those from control shRNA-transfected cells, indicating that Fzd7 knockdown inhibited tumor growth (Fig. 3a-b). Moreover, Fzd7 knockdown delayed the appearance of xenograft tumors (Fig. 3c). Tumors derived from FZD7 shRNA-transfected cells exhibited less Ki67- positive cells but more Cleaved caspase-3-positive cells (Fig. 3d). Macro-metastasis on lung surface was found in 6 of 8 control mice, and in 3 of 8 mice inoculated with FZD7 shRNA-transfected cells (Fig. 3e). In mice without macro-metastasis, micro-metastasis existed in 2 control mice and 1 mouse inoculated with FZD7 shRNAtransfected cells (Fig. 3f).

\section{Fzd7 modulates non-canonical Wnt pathway}

We next inquired the potential Wnt ligand binding to Fzd7. Intriguingly, a positive correlation of FZD7 with WNT5, especially WNT5B, was identified in both BC cell lines and BC tissues (Fig. 4a), suggesting Fzd7 might initiate non-canonical Wnt pathways. Subsequent Co-IP test confirmed the binding of Wnt5a/b to Fzd7 in both MDA-MB-231 cells and Hs578T cells (Fig. 4b-c). Furthermore, Fzd7 knockdown reduced expression of Wnt5a/b in MDA-MB-231 cells (Fig. 4d). In contrast, Fzd7 upregulation induced expression of Wnt5a/b in MCF7 cells (Fig. 4e). Loss and gain of Fzd7 affected expression of several intracellular oncogenic molecules including phosphorylated Stat3 (p-Stat3), Smad3 and Yes-associated protein 1 (Yap1) (Fig. 4d-e). Similar to Fzd7 knockdown, Wnt5b knockdown downregulated these molecules in MDA-MB-231 cells and Hs578T cells (Fig. 4f-g).

\section{Wnt5b is involved in BC cell mesenchymal phenotype and stemness}

Since Wnt5b was a potential ligand for Fzd7, we subsequently determined the role of Wnt5b in BC cell mesenchymal phenotype and stemness. Interrogation of databases showed that WNT5B, similar to FZD7, was positively associated with mesenchymal-related genes, whereas negatively associated with epithelial-related genes (Supplementary file 1: Fig. S6A-B). Wnt5b knockdown reduced expression of Vimentin, Slug and Zeb1 in BC cells (Fig. 5a-b, Supplementary file 1: Fig. S6C-D). Moreover, Wnt5b knockdown suppressed BC cell migration and invasion (Fig. 5c-d). Interrogation of databases also showed that WNT5B was associated with the panel of stemness-related genes (Fig. 5e-f). Consistently, Wnt5b knockdown impaired mammosphere formation capacity, reduced the fraction of $\mathrm{Lgr}^{+}$subpopulation and suppressed expression of CD44 in BC cells (Fig. 5g-h, Supplementary file 1: Fig. S7A-C).

\section{Col6a1 is implicated in Fzd7 signaling}

It was demonstrated that collagen signaling contributed to $\mathrm{BC}$ stemness and metastasis [19-21]. By interrogating databases, we identified the implication of Col6al in Fzd7 signaling. It was revealed that COL6A1 was associated with FZD7 and WNT5B in both BC cell lines and BC tissues (Fig. 6a-C). Immunohistochemistry detection revealed that Fzd7 and Col6a1 were expressed in a similar pattern 


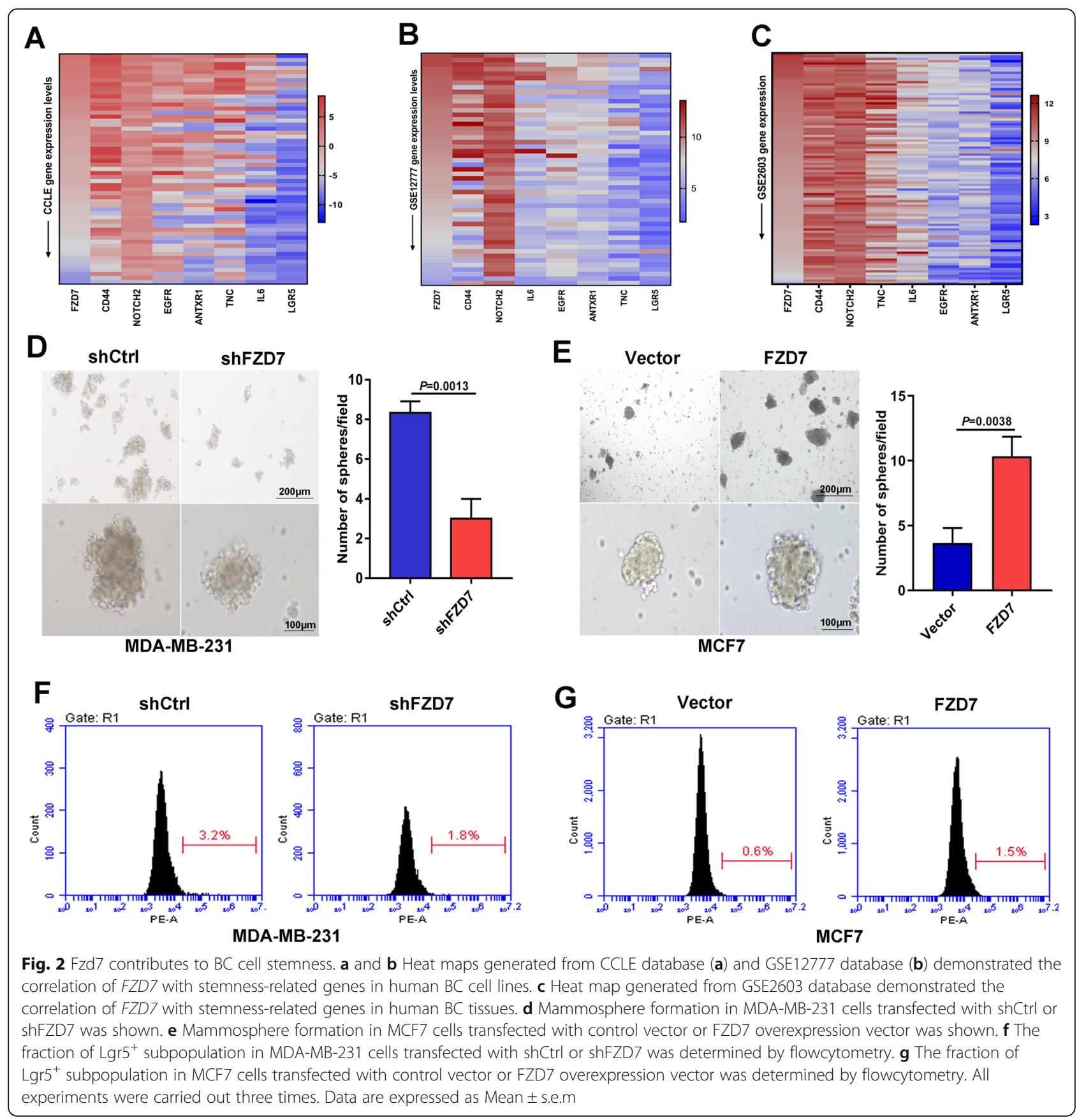

in $\mathrm{BC}$ tissues (Fig. 6d). Expression of Col6a1 was downregulated in MDA-MB-231 cells with either Fzd7 knockdown or Wnt5b knockdown (Fig. 6e-f). Intriguingly, Col6a1 knockdown inhibited expression of Wnt5a/b in BC cells (Fig. 6g-h). Col6a1 knockdown also reduced expression of p-Stat3, Smad3 and Yap1 (Fig. 6g-h).

\section{Col6a1 maintains $\mathrm{BC}$ cell mesenchymal phenotype and stemness}

We finally assessed the role of Col6a1 in $\mathrm{BC}$ cell mesenchymal phenotype and stemness. Interrogation of databases showed that COL6A1 was positively associated with mesenchymal-related genes, whereas negatively associated with epithelial-related genes (Supplementary file 1: Fig. S8A-B). Col6a1 knockdown reduced expression of Vimentin, Slug and Zeb1 in BC cells (Fig. 7a-b, Supplementary file 1: Fig. S8C-D). Col6a1 knockdown inhibited BC cell migration and invasion (Fig. 7c-d). Interrogation of databases further showed that COL6A1 was also associated with the panel of stemness-related genes (Supplementary file 1: Fig. S8E-F). As expected, Col6a1 knockdown impaired mammosphere formation 


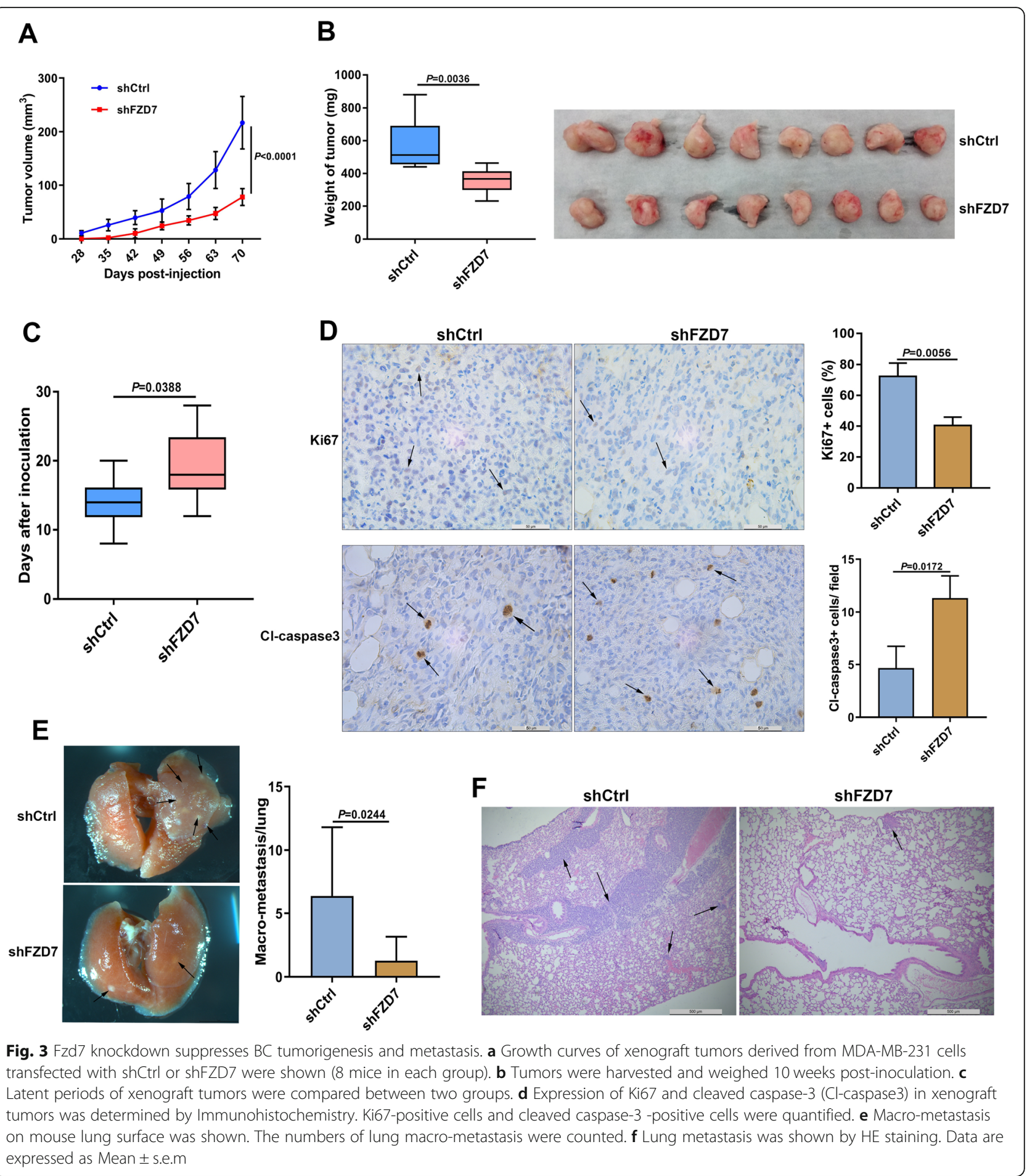

capacity of BC cells (Fig. 7e-f). Moreover, Col6a1 knockdown reduced the fraction of $\mathrm{Lgr}^{+}$subpopulation (Fig. 7g-h).

\section{Discussion}

MMTV-Wnt1, MMTV-Wnt10b and MMTV-ßcat $\Delta N$ transgenic mice have clearly demonstrated that canonical Wnt/ $\beta$-catenin pathway has the capacity to induce BC development [22]. In human BC, Wnt10b, but not Wnt1, as well as Fzd7 and Wnt co-receptor LDL receptor-related proteins 6 (LRP6) was overexpressed in TNBC, with activation of $\beta$-catenin pathway [7, 23-25]. However, our study revealed that Fzd7-mediated noncanonical Wnt pathway contributed to BC EMT and 


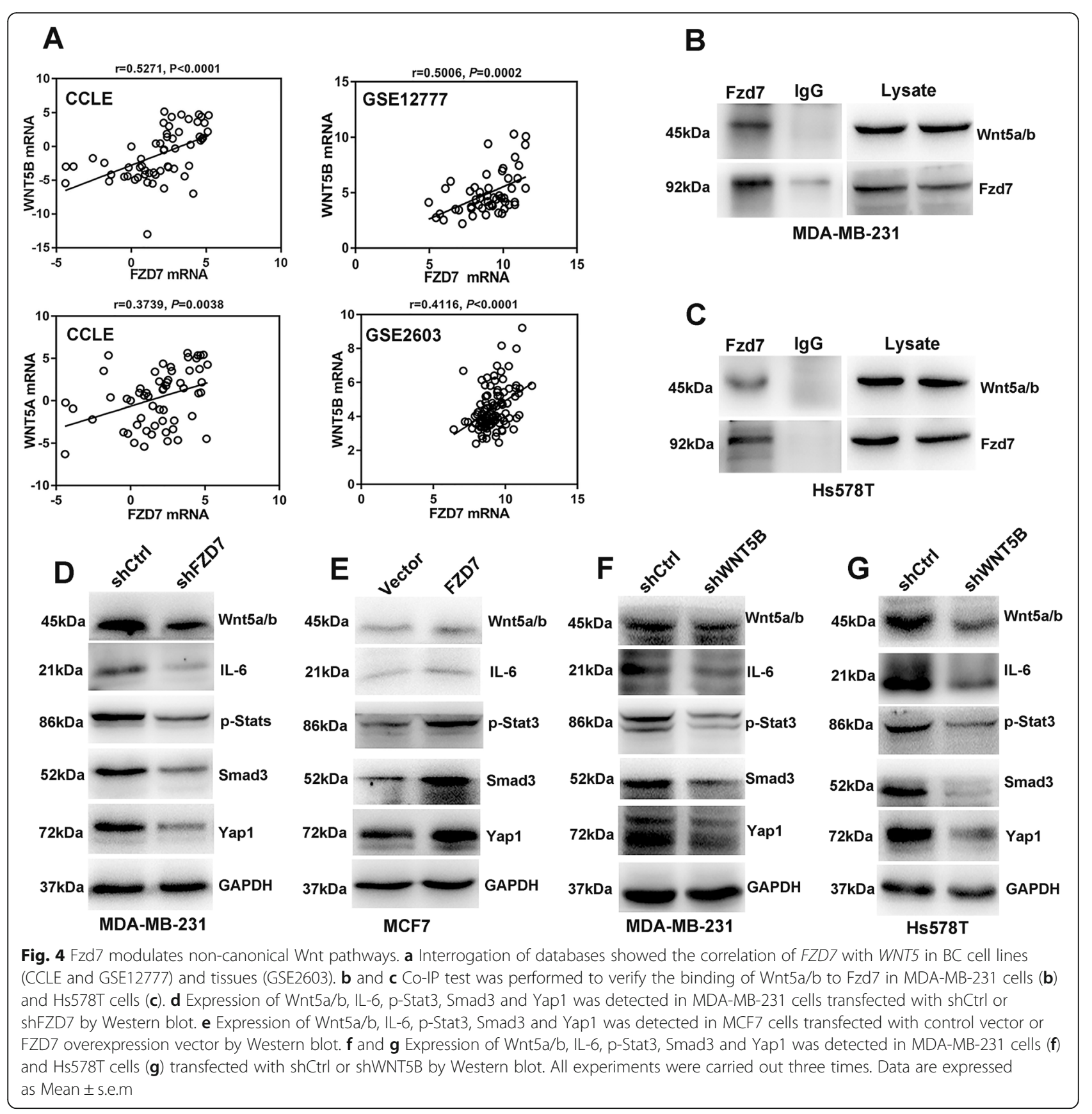

stemness. Intriguingly, both $\mathrm{Wnt} 5 \mathrm{a} / \mathrm{b}$ and Fzd7 were overexpressed in MMTV-Wnt1 tumors, suggesting that non-canonical Fzd7 pathway was involved in Wnt1induced $\mathrm{BC}$ [26]. In $\mathrm{Her}^{+}$metastasis-initiating cells, both canonical and non-canonical Wnt ligands were responsible for EMT-like program and early dissemination [27]. Therefore, we conclude that Fzd7 may mediate both $\beta$-catenin-dependent and $\beta$-catenin-independent pathways in human $\mathrm{BC}$.

Interrogation of databases indicated that Fzd7-Wnt5b pathway cross-talked with oncogenic pathways such as
IL-6, EGFR, NOTCH and TGF- $\beta 1$. We further revealed that Fzd7-Wnt5b signaling modulated several key intracellular molecules involved in these pathways including p-Stat3, Smad3 and Yap1. These findings uncovered the molecular mechanism underlying Fzd7-induced EMT and stemness. Studies have shown that IL-6, EGFR, $\mathrm{NOTCH}$ and TGF- $\beta 1$ form an oncogenic signal network in cancer initiation and progression [15, 17, 28-30]. Our study added non-canonical Fzd7 and Wnt5b into this network. Notably, Fzd7 signaling modulated Wnt5a/b expression, forming an autocrine positive feedback. 

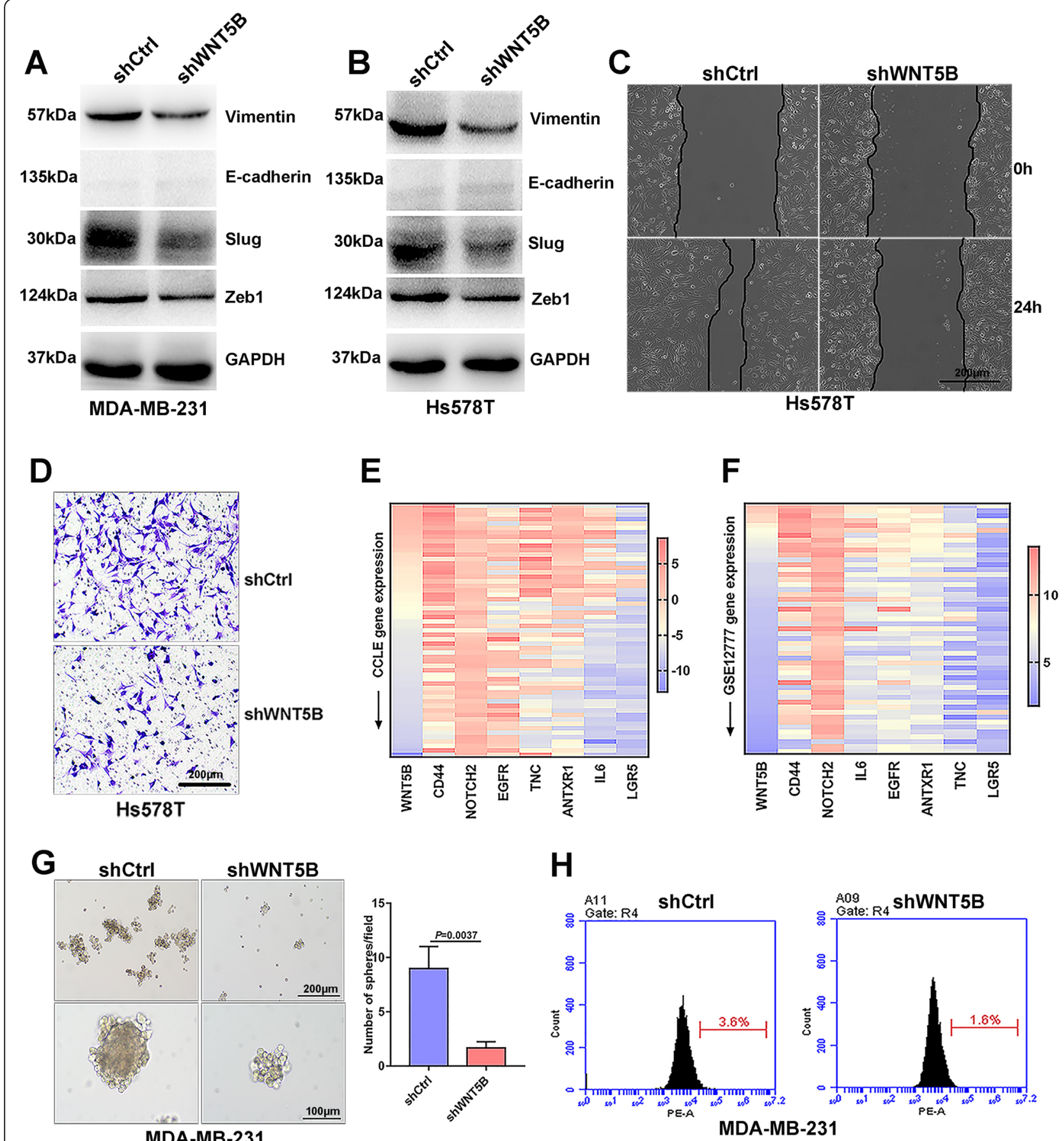

Fig. 5 Wnt5b is involved in BC cell mesenchymal phenotype and stemness. $\mathbf{a}$ and $\mathbf{b}$ Expression of Vimentin, E-cadherin, Slug and Zeb1 was detected in MDA-MB-231 cells (a) and Hs578T cells (b) transfected with shCtrl or shWNT5B by Western blot. $\mathbf{c}$ and $\mathbf{d}$ Migration (c) and invasion (d) of Hs578T cells transfected with shCtrl or shWNT5B were analyzed by Wound healing or Transwell. e and $\mathbf{f}$ Heat map generated from CCLE database (e) and GSE12777 database ( $\mathbf{f}$ ) demonstrated the correlation of WNT5B with stemness-related genes in human BC cell lines. $\mathbf{g}$ Mammosphere formation in MDA-MB-231 cells transfected with shCtrl or shWNT5B was shown. $\mathbf{h}$ The fraction of Lgr5 $5^{+}$subpopulation in MDA-MB-231 cells transfected with shCtrl or shWNT5B was determined by flowcytometry. All experiments were carried out three times. Data are expressed as Mean \pm s.e.m

Stat3, Smad3 and Yap1 might be responsible for this induction [31-33].

Lgr5 is both a marker of adult stem cells and a modulator of their activity [34]. $\operatorname{Lgr}^{+}$mammary epithelial cells were shown sufficient and necessary for postnatal mammary organogenesis [35]. Single-cell analysis demonstrated that early metastatic BC cells highly expressed EMT and stemness -associated genes including LGR5 


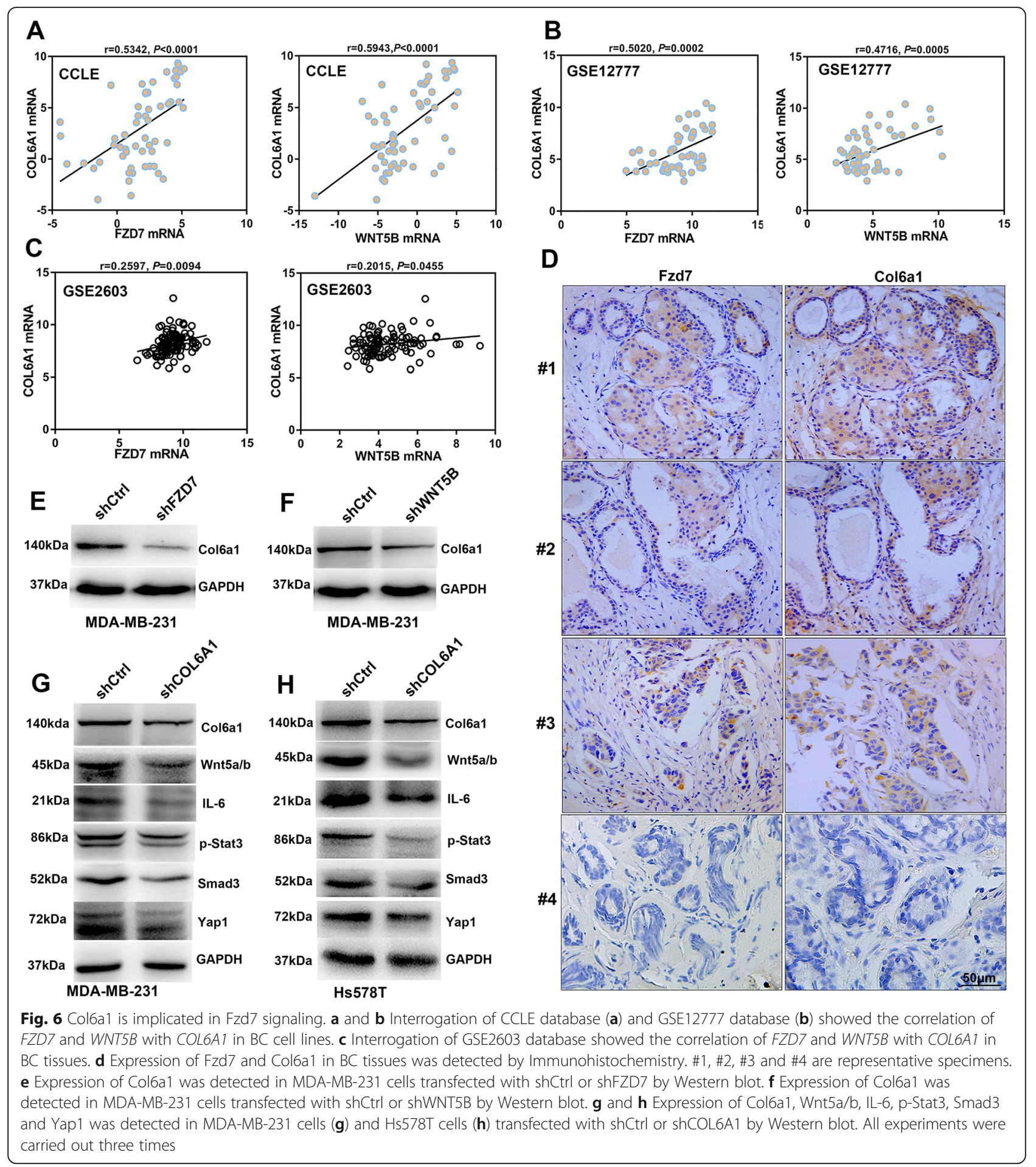

[36]. BC cell-derived Tenascin C (TNC) promoted Lgr5 expression, stemness and pulmonary metastasis [18]. Interrogation of CCLE and GSE2603 showed that FZD7 was associated with TNC and LGR5 in both BC cell lines and $\mathrm{BC}$ tissues. Furthermore, our study indicated that Fzd7 expression modulated Lgr $^{+}$subpopulation. In consistent with our finding, Fzd7 was revealed enriched in $\operatorname{Lgr}^{+}$intestinal stem cells. Deletion or blocking of Fzd7 reduced the number of stem cells or impaired the function of stem cells [37, 38]. Moreover, Wnt5a was shown to stimulate Lgr5 expression in osteoblasts [39].

Increasing evidence has indicated that collagen signaling promotes $\mathrm{BC}$ stemness and metastasis. Discoidin Domain Receptor 1 (DDR1), when exposed to its ligand 


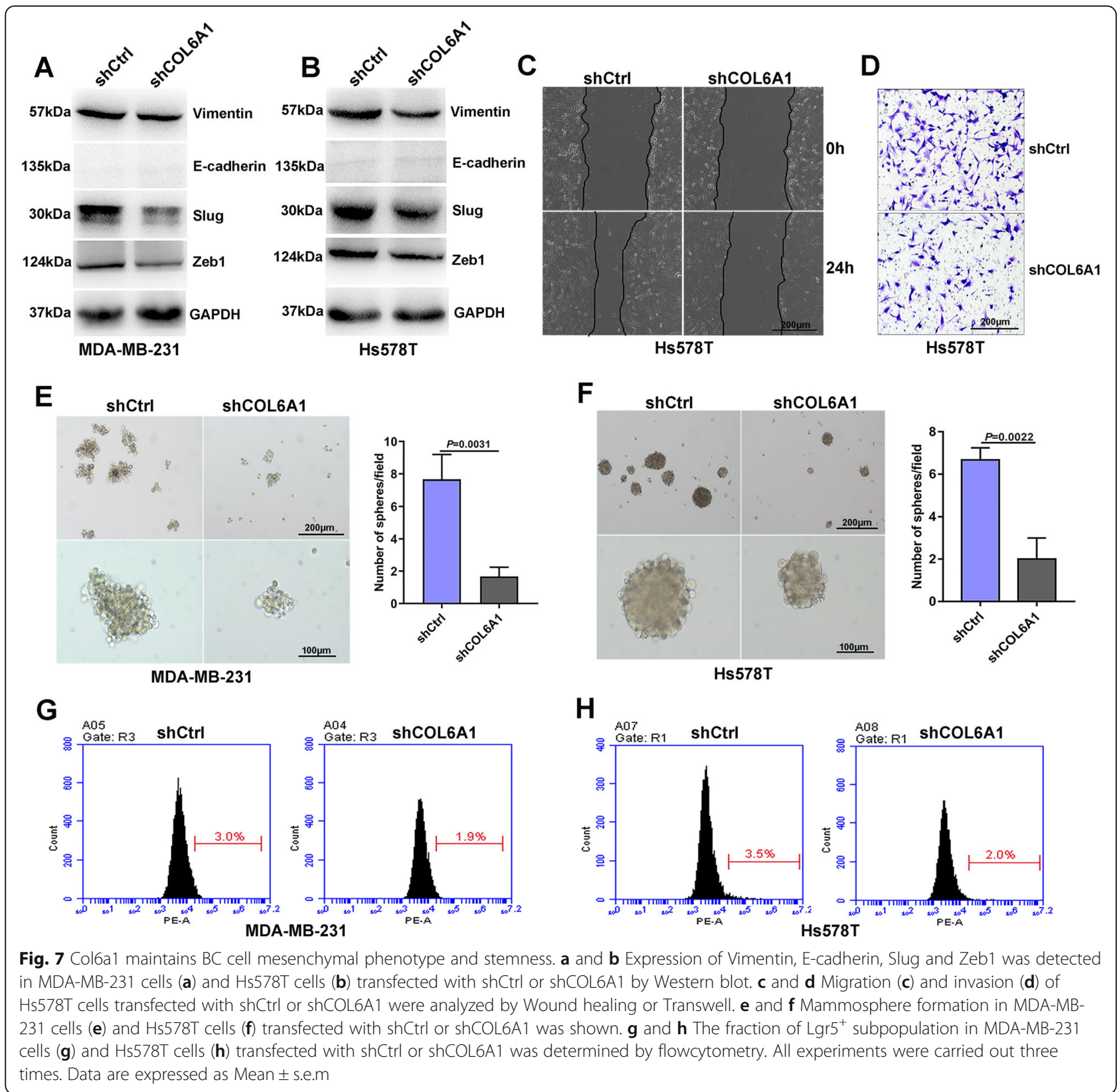

Collagen I, induced BC cell stemness and lung metastasis in a Stat3-dependent manner [21]. Interrogation of databases showed that FZD7 was closely correlated with COL1A1 (data not shown). Activation of ANTXR1 by its ligand $\mathrm{C} 5 \mathrm{a}$, a fragment of Col6a3, also increased BC cell stemness and lung metastasis [19]. Interrogation of databases showed that FZD7 was associated with ANTXR1. These observations, in combine with our finding that Fzd7-Wnt5b modulated expression of Col6a1, indicate that non-canonical Fzd7 signaling cross-talked with Collagen signaling.

\section{Conclusions}

Our study identified that Fzd7 contributed to BC cell EMT and stemness, inducing tumorigenesis and metastasis, via a non-canonical Wnt5b pathway. We also discovered that Col6a1 was implicated in Fzd7-Wnt5b signaling, and mediated Fzd7-Wnt5b -induced mesenchymal-like stemness. These findings linked non-canonical Wnt pathway to Collagen signaling in BC progression. Mechanistically, we proposed an oncogenic network consisting of non-canonical Wnt, Collagen, IL-6/Stat3, TGF- $11 /$ Smad3, EGFR and NOTCH2. 


\section{Supplementary information}

Supplementary information accompanies this paper at https://doi.org/10. 1186/s12964-020-00646-2.

Additional file 1: Supplementary Figure S1. Interrogation of CCLE database (A) and GSE12777 database (B) showed the correlation of FZD7 with TGFB1, VIM, SNAI2 (Slug) and ZEB1 in BC cell lines. Expression of VIM, CDH1, SNAI2 and ZEB1 was detected in MDA-MB-231 (C) and Hs578T (D) cells transfected with shCtrl or shFZD7 by real-time PCR. Expression of N-cadherin was detected in MDA-MB-231 (E) and Hs578T (F) cells transfected with shCtrl or shFZD7 by Western blot. All experiments were carried out three times. Data are expressed as Mean \pm s.e.m. Supplementary Figure S2. (A) Expression of Fzd7, Vimentin, E-cadherin, Slug and Zeb1 was detected in MCF7 cells transfected with control vector or FZD7 overexpression vector by Western blot. Expression of Vimentin (B) and E-cadherin (C) was detected in MCF7 cells transfected with control vector or FZD7 overexpression vector by Immunofluorescence staining. All experiments were carried out three times. Supplementary Figure S3. Migration (A) and Invasion (B) of MDA-MB-231 cells transfected with shCtrl or shFZD7 was analyzed by Wound healing and Transwell, respectively. (C) Invasion of Hs578T cells transfected with shCtrl or shFZD7 was analyzed by Transwell. (D) Migration of MCF7 cells transfected with control vector or FZD7 overexpression vector was analyzed by Wound healing. All experiments were carried out three times. Supplementary Figure S4. (A) Interrogation of CCLE database showed the correlation of FZD7 with CD44, LGR5, EGFR and NOTCH2 in BC cell lines. (B) Interrogation of GSE12777 database showed the correlation of FZD7 with CD44, EGFR and NOTCH2 in BC cell lines. (C) Interrogation of GSE2603 database showed the correlation of FZD7 with LGR5, EGFR and NOTCH2 in $B C$ tissues. Supplementary Figure S5. (A) Mammosphere formation in Hs578T cells transfected with shCtrl or shFZD7 was shown. (B) The fraction of $\mathrm{Lgr5}^{+}$subpopulation in Hs578T cells transfected with shCtrl or shFZD7 was determined by flowcytometry. (C) Expression of CD44 was detected in MDA-MB-231 cells transfected with shCtrl or shFZD7 by Western blot. (D) Expression of CD44 was detected in MCF7 cells transfected with control vector or FZD7 overexpression vector by Western blot. All experiments were carried out three times. Data are expressed as Mean \pm s.e.m. Supplementary Figure S6. Heat maps generated from CCLE database (A) and GSE12777 database (B) demonstrated the correlation of WNT5B with mesenchymal-related genes and epithelial-related genes in human BC cell lines. Expression of VIM, CDH1, SNAI2 and ZEB1 was detected in MDA-MB-231 (C) and Hs578T (D) cells transfected with shCtrl or shWNT5B by real-time PCR. All experiments were carried out three times. Data are expressed as Mean \pm s.e.m. Supplementary Figure S7. (A) Mammosphere formation in Hs578T cells transfected with shCtrl or shWNT5B was shown. (B) The fraction of $\mathrm{Lgr5}^{+}$subpopulation in Hs578T cells transfected with shCtrl or shWNT5B was determined by flowcytometry. (C) Expression of CD44 was detected in MDA-MB-231 cells transfected with shCtrl or shWNT5B by Western blot. All experiments were carried out three times. Data are expressed as Mean \pm s.e.m. Supplementary

Figure S8. Heat maps generated from CCLE database (A) and GSE12777 database (B) demonstrated the correlation of COL6A1 with mesenchymal-related genes and epithelial-related genes in human BC cell lines. Expression of VIM, CDH1, SNAI2 and ZEB1 was detected in MDA-MB-231 (C) and Hs578T (D) cells transfected with shCtrl or shCOL6A1 by real-time PCR. Heat maps generated from CCLE database (E) and GSE12777 database (F) demonstrated the correlation of COL6A1 with stemness-related genes in human BC cell lines. All experiments were carried out three times. Data are expressed as Mean \pm s.e.m.

\section{Abbreviations}

EMT: Epithelial-mesenchymal transition; BC: Breast cancer; Fzd7: Frizzled 7; pSTAT3: Phosphorylated Stat3; CRD: Cysteine-rich domain; HCC: Hepatocellular carcinoma.; TNBC: Triple-negative breast cancer; Col6a1: Collagen; type VI: Alpha 1; CCLE: Cancer Cell Line Encyclopedia; EMT-TF: EMT transcription factor; ALDH1: Aldehyde dehydrogenase 1; Yap1: Yes-associated protein 1; TNC: Tenascin C; LRP6: LDL receptor-related proteins 6; DDR1: Discoidin Domain Receptor 1

\section{Acknowledgements}

Not applicable.

\section{Authors' contributions}

PY performed the experiments and wrote the manuscript. YB and ZW conducted the experiments and contributed to the analysis of data. YS, JG, ZZ and LN performed the experiments. CZ and WW designed the project, performed the interpretation of the data, and drafted the manuscript. All authors read and approved the final manuscript.

\section{Funding}

This work is supported by a grant from Liaoning Province Science and Technology Department (2017225028).

\section{Competing interests}

The authors declare that they have no competing interests.

\section{Author details}

${ }^{1}$ Department of Pathophysiology, College of Basic Medical Science, China Medical University, Shenyang, China. '2Department of Nephrology, Shengjing Hospital, China Medical University, Shenyang, China.

Received: 19 September 2019 Accepted: 12 August 2020

Published online: 07 September 2020

\section{References}

1. Binda E, Visioli A, Giani F, Trivieri N, Palumbo O, Restelli S, et al. Wnt5a drives an invasive phenotype in human Glioblastoma stem-like cells. Cancer Res. 2017;77(4):996-1007.

2. Hayakawa Y, Ariyama H, Stancikova J, Sakitani K, Asfaha S, Renz BW, et al. Mist1 expressing gastric stem cells maintain the Normal and neoplastic gastric epithelium and are supported by a perivascular stem cell niche. Cancer Cell. 2015;28(6):800-14.

3. Chen YC, Gonzalez ME, Burman B, Zhao X, Anwar T, Tran M, et al. Mesenchymal Stem/Stromal Cell Engulfment Reveals Metastatic Advantage in Breast Cancer. Cell Reports. 2019:27(13):3916-26.e5.

4. Merle P, de la Monte S, Kim M, Herrmann M, Tanaka S, Von Dem Bussche A, et al. Functional consequences of frizzled-7 receptor overexpression in human hepatocellular carcinoma. Gastroenterology. 2004;127(4):1110-22.

5. Merle P, Kim M, Herrmann M, Gupte A, Lefrancois L, Califano S, et al. Oncogenic role of the frizzled-7/beta-catenin pathway in hepatocellular carcinoma. J Hepatol. 2005;43(5):854-62.

6. Kim M, Lee HC, Tsedensodnom O, Hartley R, Lim YS, Yu E, et al. Functional interaction between Wnt3 and Frizzled-7 leads to activation of the Wnt/ beta-catenin signaling pathway in hepatocellular carcinoma cells. J Hepatol. 2008;48(5):780-91.

7. Yang L, Wu X, Wang Y, Zhang K, Wu J, Yuan YC, et al. FZD7 has a critical role in cell proliferation in triple negative breast cancer. Oncogene. 2011; 30(43):4437-46.

8. King TD, Suto MJ, Li Y. The Wnt/beta-catenin signaling pathway: a potential therapeutic target in the treatment of triple negative breast cancer. J Cell Biochem. 2012;113(1):13-8.

9. Anastas JN, Kulikauskas RM, Tamir T, Rizos H, Long GV, von Euw EM, et al. WNT5A enhances resistance of melanoma cells to targeted BRAF inhibitors. J Clin Invest. 2014;124(7):2877-90

10. Flanagan DJ, Barker N, Costanzo NSD, Mason EA, Gurney A, Meniel VS, et al. Frizzled-7 Is Required for Wnt Signaling in Gastric Tumors with and Without Apc Mutations. Cancer Res. 2019;79(5):970-81.

11. Liu S, Cong Y, Wang D, Sun Y, Deng L, Liu Y, et al. Breast cancer stem cells transition between epithelial and mesenchymal states reflective of their normal counterparts. Stem Cell Reports. 2014;2(1):78-91.

12. Guen VJ, Chavarria TE, Kroger C, Ye X, Weinberg RA, Lees JA. EMT programs promote basal mammary stem cell and tumor-initiating cell stemness by inducing primary ciliogenesis and hedgehog signaling. Proc Natl Acad Sci U S A. 2017:114(49):E10532-e9.

13. Mani SA, Guo W, Liao MJ, Eaton EN, Ayyanan A, Zhou AY, et al. The epithelial-mesenchymal transition generates cells with properties of stem cells. Cell. 2008;133(4):704-15.

14. Marotta LL, Almendro V, Marusyk A, Shipitsin M, Schemme J, Walker SR, et al. The JAK2/STAT3 signaling pathway is required for growth of CD44(+ 
)CD24(-) stem cell-like breast cancer cells in human tumors. J Clin Invest. 2011;121(7):2723-35.

15. Ibrahim SA, Gadalla R, El-Ghonaimy EA, Samir O, Mohamed HT, Hassan H, et al. Syndecan-1 is a novel molecular marker for triple negative inflammatory breast cancer and modulates the cancer stem cell phenotype via the IL-6/STAT3, Notch and EGFR signaling pathways. Mol Cancer. 2017; 16(1):57.

16. Yen WC, Fischer MM, Axelrod F, Bond C, Cain J, Cancilla B, et al. Targeting Notch signaling with a Notch2/Notch3 antagonist (tarextumab) inhibits tumor growth and decreases tumor-initiating cell frequency. Clin Cancer Res. 2015;21(9):2084-95.

17. Yeo SK, Wen J, Chen S, Guan JL. Autophagy differentially regulates distinct breast Cancer stem-like cells in murine models via EGFR/Stat3 and Tgfbeta/ Smad signaling. Cancer Res. 2016;76(11):3397-410.

18. Oskarsson T, Acharyya S, Zhang XH, Vanharanta S, Tavazoie SF, Morris PG, et al. Breast cancer cells produce tenascin $C$ as a metastatic niche component to colonize the lungs. Nat Med. 2011:17(7):867-74.

19. Chen D, Bhat-Nakshatri P, Goswami C, Badve S, Nakshatri H. ANTXR1, a stem cell-enriched functional biomarker, connects collagen signaling to cancer stem-like cells and metastasis in breast cancer. Cancer Res. 2013;73(18): 5821-33.

20. Zhang H, Fredericks T, Xiong G, Qi Y, Rychahou PG, Li JD, et al. Membrane associated collagen XIII promotes cancer metastasis and enhances anoikis resistance. Breast Cancer Res. 2018;20(1):116.

21. Gao H, Chakraborty G, Zhang Z, Akalay I, Gadiya M, Gao Y, et al. Multi-organ site metastatic reactivation mediated by non-canonical Discoidin domain receptor 1 signaling. Cell. 2016;166(1):47-62.

22. Yin P, Wang W, Zhang Z, Bai Y, Gao J, Zhao C. Wnt signaling in human and mouse breast cancer: Focusing on Wnt ligands, receptors and antagonists. Cancer Sci. 2018;109(11):3368-75.

23. Meyers SL, O'Brien MT, Smith T, Dudley JP. Analysis of the int-1, int-2, c-myc, and neu oncogenes in human breast carcinomas. Cancer Res. 1990;50(18): 5911-8.

24. Wend $\mathrm{P}$, Runke $\mathrm{S}$, Wend $\mathrm{K}$, Anchondo $\mathrm{B}$, Yesayan $\mathrm{M}$, Jardon $\mathrm{M}$, et al. WNT10B/beta-catenin signalling induces HMGA2 and proliferation in metastatic triple-negative breast cancer. EMBO Mol Med. 2013;5(2):264-79.

25. Liu CC, Prior J, Piwnica-Worms D, Bu G. LRP6 overexpression defines a class of breast cancer subtype and is a target for therapy. Proc Natl Acad Sci U S A. 2010;107(11):5136-41.

26. Huang S, Li Y, Chen Y, Podsypanina K, Chamorro M, Olshen AB, et al. Changes in gene expression during the development of mammary tumors in MMTV-Wnt-1 transgenic mice. Genome Biol. 2005;6(10):R84.

27. Harper KL, Sosa MS, Entenberg D, Hosseini H, Cheung JF, Nobre R, et al. Mechanism of early dissemination and metastasis in Her2(+) mammary cancer. Nature. 2016;540(7634):588-92.

28. Zhao $K$, Wang $Q$, Wang $Y$, Huang $K$, Yang $C$, Li Y, et al. EGFR/c-myc axis regulates TGFbeta/hippo/Notch pathway via epigenetic silencing miR-524 in gliomas. Cancer Lett. 2017:406:12-21.

29. Lee SY, Jeong EK, Ju MK, Jeon HM, Kim MY, Kim CH, et al. Induction of metastasis, cancer stem cell phenotype, and oncogenic metabolism in cancer cells by ionizing radiation. Mol Cancer. 2017;16(1):10.

30. Kannan A, Krishnan A, Ali M, Subramaniam S, Halagowder D, Sivasithamparam ND. Caveolin-1 promotes gastric cancer progression by up-regulating epithelial to mesenchymal transition by crosstalk of signalling mechanisms under hypoxic condition. Eur I Cancer (Oxford, England : 1990). 2014;50(1):204-15.

31. Katoh M, Katoh M. STAT3-induced WNT5A signaling loop in embryonic stem cells, adult normal tissues, chronic persistent inflammation, rheumatoid arthritis and cancer (review). Int J Mol Med. 2007;19(2):273-8.

32. Katoh M, Katoh M. Transcriptional mechanisms of WNT5A based on NFkappaB, hedgehog, TGFbeta, and Notch signaling cascades. Int J Mol Med. 2009;23(6):763-9.

33. Park HW, Kim YC, Yu B, Moroishi T, Mo JS, Plouffe SW, et al. Alternative Wnt signaling activates YAP/TAZ. Cell. 2015;162(4):780-94.

34. Leung C, Tan SH, Barker N. Recent advances in Lgr5(+) stem cell research. Trends Cell Biol. 2018;28(5):380-91.

35. Plaks V, Brenot A, Lawson DA, Linnemann JR, Van Kappel EC, Wong KC, et al. Lgr5-expressing cells are sufficient and necessary for postnatal mammary gland organogenesis. Cell Rep. 2013;3(1):70-8.
36. Lawson DA, Bhakta NR, Kessenbrock K, Prummel KD, Yu Y, Takai K, et al. Single-cell analysis reveals a stem-cell program in human metastatic breast cancer cells. Nature. 2015;526(7571):131-5.

37. Flanagan DJ, Phesse TJ, Barker N, Schwab RH, Amin N, Malaterre J, et al. Frizzled7 functions as a Wnt receptor in intestinal epithelial $\operatorname{Lgr} 5(+)$ stem cells. Stem Cell Reports. 2015;4(5):759-67.

38. Nile AH, de Sousa EMF, Mukund S, Piskol R, Hansen S, Zhou L, et al. A selective peptide inhibitor of Frizzled 7 receptors disrupts intestinal stem cells. Nat Chem Biol. 2018;14(6):582-90.

39. Martineau X, Abed E, Martel-Pelletier J, Pelletier JP, Lajeunesse D. Alteration of Wnt5a expression and of the non-canonical Wnt/PCP and Wnt/PKC-Ca2+ pathways in human osteoarthritis osteoblasts. PLoS One. 2017;12(8): e0180711.

\section{Publisher's Note}

Springer Nature remains neutral with regard to jurisdictional claims in published maps and institutional affiliations.

\section{Ready to submit your research? Choose BMC and benefit from:}

- fast, convenient online submission

- thorough peer review by experienced researchers in your field

- rapid publication on acceptance

- support for research data, including large and complex data types

- gold Open Access which fosters wider collaboration and increased citations

- maximum visibility for your research: over $100 \mathrm{M}$ website views per year

At BMC, research is always in progress.

Learn more biomedcentral.com/submissions 